\title{
NANOINDENTATION SIMULATION ON MECHANICAL BEHAVIOR OF NANOCRYSTALLINE Ni
}

\author{
QIHENG TANG* and TIANYONG YANG \\ State Key Laboratory of Nonlinear Mechanics, Institute of Mechanics, \\ Chinese Academy of Sciences, Beijing 100190, P. R. China \\ State Key Laboratory of Explosion Science and Technology, Beijing Institute of Technology, \\ Beijing 100081, P. R. China \\ *qhtang@lnm.imech.ac.cn
}

Received 28 September 2009

Revised 25 November 2009

\begin{abstract}
The indentation simulation of the nanocrystalline Ni is carried out by molecular dynamics technique (MD) to study the mechanical behavior at nanometer scales. The sphere indenter is used, and simulation sample with three grains and two grain boundaries is adopted. The strength of nanocrystalline is studied as indenter is set at grain boundary and grain, respectively. Some defects such as dislocations or slipping deformation are observed. It is found that dislocations are emitted from the grain boundary or the sample surface. The temperature distribution of local region around indenter is analyzed and it can explain our MD simulation results.
\end{abstract}

Keywords: Molecular dynamics simulation; indentation; nanocrystalline; defect evolution.

\section{Introduction}

Investigation of deformation mechanism on nanometer materials is a hot topic. Indentation is a remarkably flexible mechanical test due to its relative experimental simplicity. ${ }^{1,2} \mathrm{Li}$ et al. performed nanoindentation tests on silver nanowires ${ }^{1}$ and $\mathrm{ZnS}$ nanobelts. $^{2}$

The merit of AFM-based lithography techniques is that AFM can exert a very small vertical force on the workpiece surface, which leads to a depth of cut of several nanometers, and a wealth of information of deformation is obtained. Unfortunately, the cutting mechanism at the atomic scale is not well understood, and the experimental picture cannot display the atomic process involved in dislocation nucleation and micro defects evolution.

Landman et al. ${ }^{3}$ and Hoover et $a l .{ }^{4}$ may be the ones who firstly performed the atomic-level simulations of nanoindentation ${ }^{5}$ and they are interested in using

\footnotetext{
*Corresponding author.
} 
these simulations to probe bulk elastic-plastic responses. ${ }^{6}$ A large body of work by simulating nanoindentation appeared, some remarkable works should be mentioned. Kelchner et al. observed dislocation generated under $\mathrm{Au}(111)$ surface after indentation by a smooth sphere. ${ }^{7}$ They paid attention to characterizing the bulk atomic environment for defect visualization, and their calculation showed that the initial dislocations may be nucleated inside bulk near the spherical indenter, but cannot be generated from the surface. This was later verified by Gouldstone et al. in experiment. ${ }^{8}$ It means that the surface is not dislocation source uniquely. Combining experiments with atomistic simulation, Li et al. ${ }^{9}$ proposed a soft-phonon criterion to address the stress condition required for homogeneous nucleation at temperature of $0 \mathrm{~K}$, and it activated ones to probe conditions of defects generated and evolution. ${ }^{10,11}$

The responses of grain boundary (GB) under an indenter have been focused for a long time. Simulation of GB-aided partial dislocation nucleation was given by Ma and Yang, ${ }^{12}$ and the results showed that the threshold stress for partial dislocation to heterogeneously nucleate is lower than that to homogeneously nucleate inside the perfect lattice. The deformation of nanocrystalline Au with grain diameters 5 and $12 \mathrm{~nm}$ was investigated by Feichtinger et al. ${ }^{13}$ It is found that GB may act as efficient sinks for dislocation nucleation below the spherical indenter and that intergranular sliding occurs. The effect of grain size on material strength was studied by Van Vliet et al. ${ }^{14}$ They adopted nanoindentation as a means to introduce localized deformation, and quantified critical conditions for defect nucleation. In the strengthening regime, the primary deformation mechanism was discrete dislocation emission from grain boundary regions, whereas in the weakening regime, the primary deformation mechanism was grain migration.

In this paper, a MD simulation under the canonical ensemble (NVT) is applied to study the dislocations nucleation and defects evolution of nanocrystalline (nc) $\mathrm{Ni}$ during the nanoindentation process. Special attention is paid to the comparison of mechanical behaviors as the sphere indenter is at grain boundary and at inside grain.

\section{Computation and Modeling}

\subsection{Interatomic potential}

It is known that the accuracy of MD simulations is dictated by the accuracy of the interatomic potential being used. The embedded atom method (EAM) to describe potential for FCC $\mathrm{Ni}^{15}$ is adopted. In this context, the total energy of the system is given by

$$
E_{T}=\sum_{i=1}^{N} F_{i}\left(\rho_{i}\right)+\frac{1}{2} \sum_{i j, i \neq j} \phi_{i j}\left(r_{i j}\right),
$$

where $\phi_{i j}\left(r_{i j}\right)$ is the pair interaction and $r_{i j}$ is the distance between atoms $i$ and $j$, $F_{i}$ is the embedding function and $\rho_{i}$ is the electronic density at the site of atom $i$. Most simulation results show that the EAM potential is valid for metallic materials. 
All parameters for the interatomic potential of Ni can be found. ${ }^{15}$ The interaction between $\mathrm{Ni}$ atoms and the diamond pin tool is modeled by the repulsive potential $V_{p}(r),{ }^{16} V_{p}(r)=A r^{-n}$, where $A$ and $n$ are material parameters. In this work, $A$ was chosen as $10 \mathrm{nN}$ (Angstrom) ${ }^{4}$ and $n$ as 3 . The experiment shows that the $\mathrm{Ni}$ hardness is about $3.4 \mathrm{GPa}$ and of diamond is $78.96 \mathrm{GPa}^{14}$; one can assume that the diamond tip does not deform during the process of indentation.

The components of the stress tensor ${ }^{17}$ associated with an atom are defined as

$$
\sigma_{k l}=\frac{1}{\Omega_{0}}\left(-\frac{p_{k} p_{l}}{m}+\sum_{j \neq i} \frac{1}{2} \frac{\partial \phi}{\partial r} \frac{r_{i j}^{k} r_{i j}^{l}}{r_{i j}}\right),
$$

where $\sigma_{k l}$ is the stress component, $p$ is the atomic momentum, $m$ is the atomic mass, $r_{i j}^{k}$ and $r_{i j}^{l}$ are the $k$ th, $l$ th components of $r_{i j}$, and $\Omega_{0}$ is the volume of an atom.

\subsection{Models of nanocrystalline $\mathrm{Ni}$}

The initial simulated nc is generated using a Voronoi construction, ${ }^{18}$ it contains three grains and two grain boundaries, about 200,000 atoms. A MD simulation with NPT technique at room temperature is carried out to relax any possible high potential energy configuration, and the Parrinello-Rahman approach ${ }^{19}$ is applied with periodic boundary conditions. The crystal orientation of G2 is along $X[100]$, $Y[010]$, and $Z[001]$. Figure 1 displays the schematic of nc Ni indentation model.

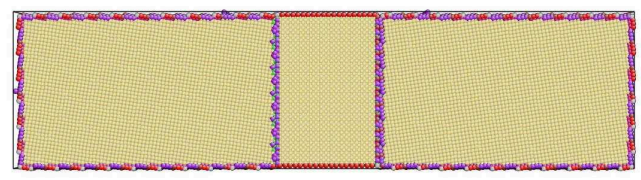

(a)

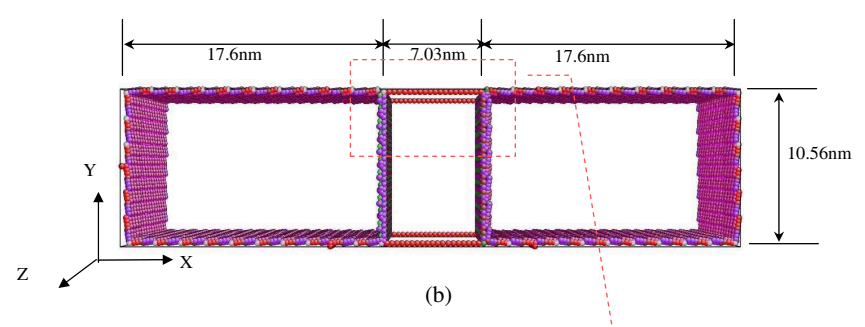

(b)

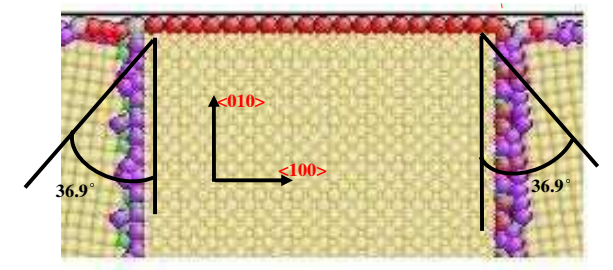

(c)

Fig. 1. Schematic of nanometer Ni indentation model: (a) atomic configuration before NPT simulation, (b) and (c) atomic configuration of surface and grain boundary before NPT simulation. 
Figure 1(a) shows atomic configuration before NPT simulation; after removing the perfect atoms, the atoms with nonperfect lattice are plotted in Figs. 1(b) and 1(c). Figure 2 displays the atomic configuration both surface and grain boundary structure after NPT MD simulation. Figure 3 displays two indentation models of nc Ni.

The MD simulations are performed under the NVT ensemble. Initial velocities of atoms are specified based on the Maxwellian distribution corresponding to $T=$ $300 \mathrm{~K}$, and the magnitudes may be adjusted so as to keep temperature constant in

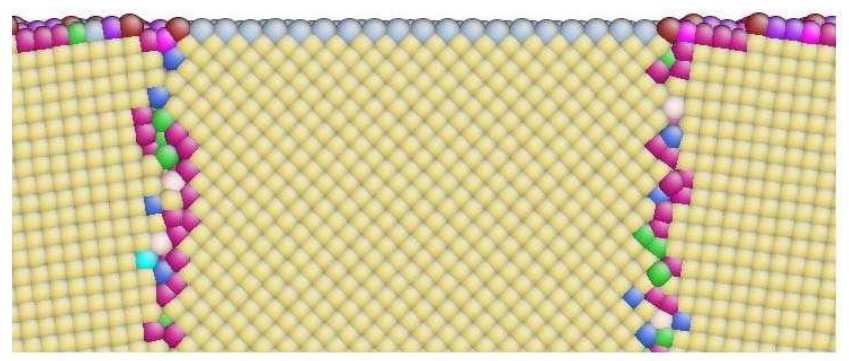

(a)

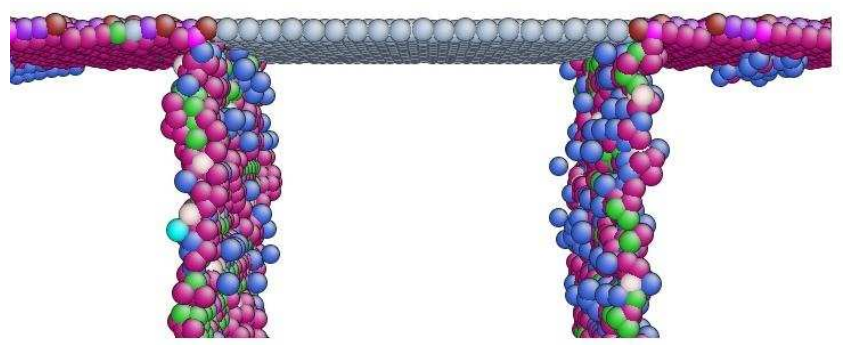

(b)

Fig. 2. Atomic configuration after NPT simulation: (a) atomic configuration in $x z$ profile, (b) atomic configuration in both surface and grain boundary.

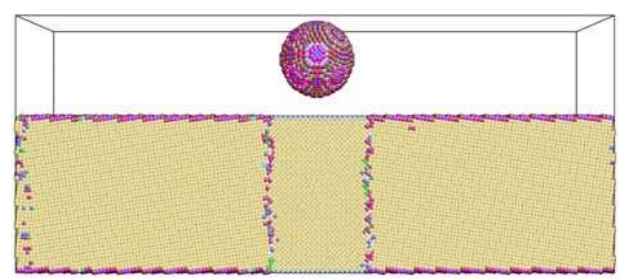

(a)

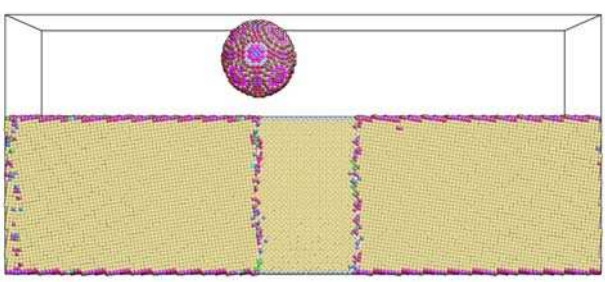

(b)

Fig. 3. Indentation models of nanocrystalline $\mathrm{Ni}$ : (a) indenter set at grain boundary GB1, (b) indenter set at grain G2. 
the system according to the Nose-Hoover thermostat. ${ }^{20,21}$ The time integration of motion is performed by using the velocity-Verlet algorithm. ${ }^{22}$

\section{Results and Discussion}

For simplification in the following analysis, three grains are labeled as G1, G2, and G3 from left to right, and two grain boundaries are labeled as GB1 and GB2, respectively. Two examples are performed with the same loading rate and temperature, but the sphere indenter is set at different sites of nc sample. The loading rate is at $0.00704 \mathrm{~nm} / \mathrm{ps}$ and temperature is $T=300 \mathrm{~K}$. One example is to set sphere indenter at grain G2, and other example is to set indenter at grain GB1, see Fig. 3. Figure 4 shows the comparison of curves of thrust forces $F_{z}$ versus indentation depth. The thrust force $F_{z}$ increases with the indentation depth. The indentation depth is within $4 \mathrm{~nm}$, the different thrust forces are obtained due to the different indentation sites. The thrust force of indentation site set at GB1 is a little smaller than that of the indentation site at G2. It implies that the strength of grain boundary is weaker than that of grain at the initial stage of indentation. But the difference disappears as indentation depth is near $4 \mathrm{~nm}$. It implies that there may be some transition of crystal structure during the indentation.

Figure 5 displays four atom configurations of indentation. As the indenter presses into nc $\mathrm{Ni}$ at $\mathrm{G} 2$ and reaches at $1.0 \mathrm{~nm}$, the shear deformation of the lattice at the left upper of G2 is observed along $45^{\circ}$ with respect to the loading direction, and it originated from surface. The mechanical analysis pointed out that as a sample is compressed under uniaxial load, the shear stress is bigger along $45^{\circ}$ with respect to the loading direction, see arrow at Fig. 5(a). The shear deformation

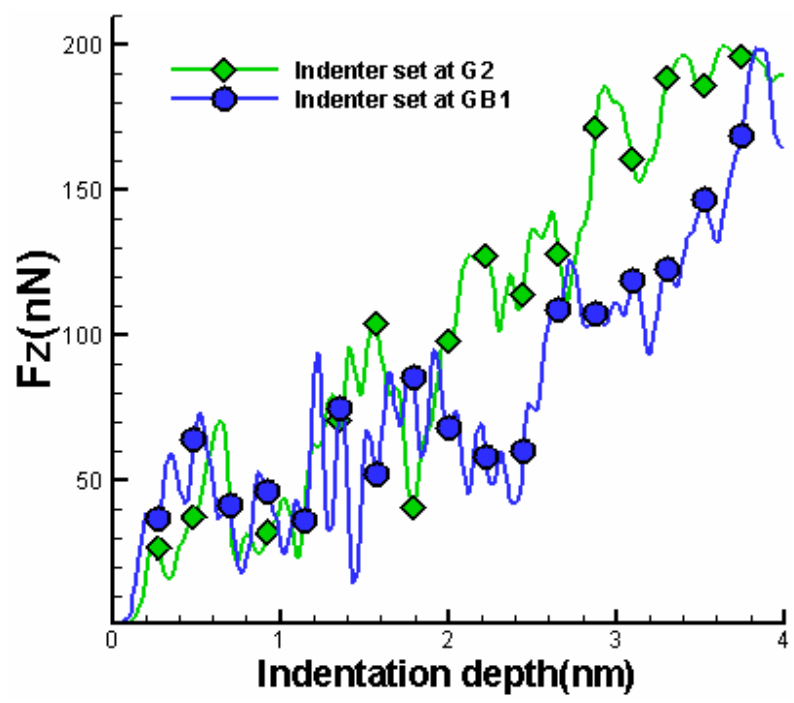

Fig. 4. Comparison of thrust force between case 1 and case 2 . 
is due to lattice relatively slipping along the slip plane. As the pin tool reaches at $2.0 \mathrm{~nm}$, the shear deformation is observed at the several slip planes, and they are originated from grain boundaries GB1 and GB2, see arrows at Fig. 5(b). Both experiments and simulations have verified that the grain boundaries are dislocation source, dislocations emitted from grain boundary leading to the plastic deformation. Both GB1 and GB2 keep the straight lines, it means that the partial dislocation emitted from grain boundary does not damage the lattice structure of grain boundary. As the applied load increases continuously and the indenter reaches at $3 \mathrm{~nm}$,

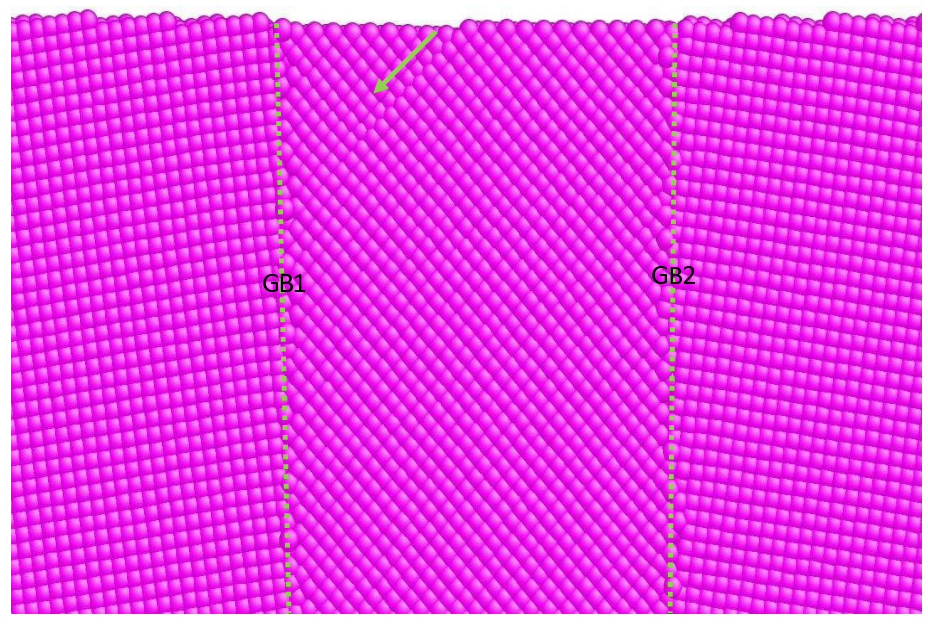

(a) $1 \mathrm{~nm}$

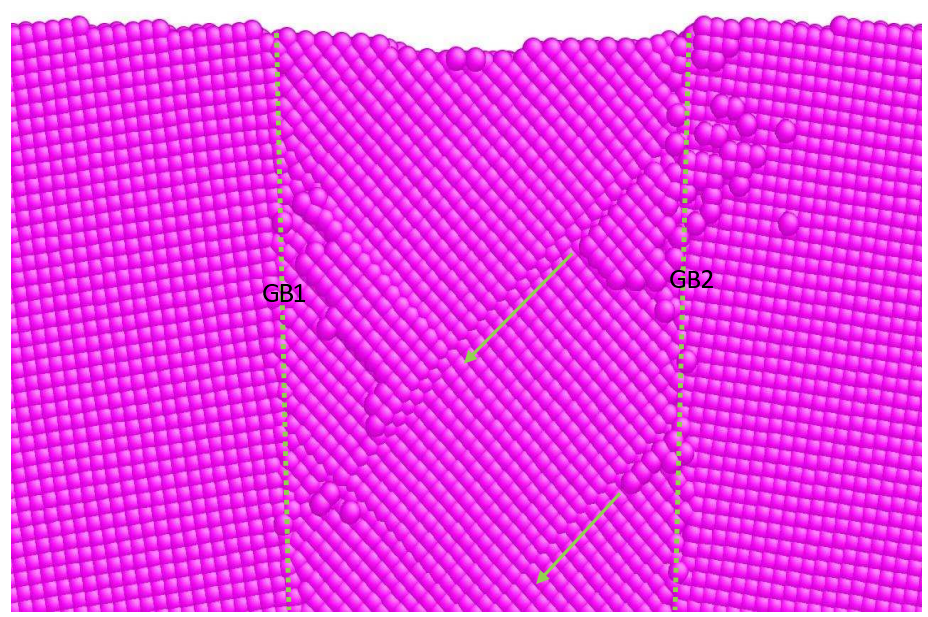

(b) $2 \mathrm{~nm}$

Fig. 5. Defects evolution during indentation for example 1: (a) $1.0 \mathrm{~nm}$, (b) $2.0 \mathrm{~nm}$, (c) $3.0 \mathrm{~nm}$, and (d) $4.0 \mathrm{~nm}$. 


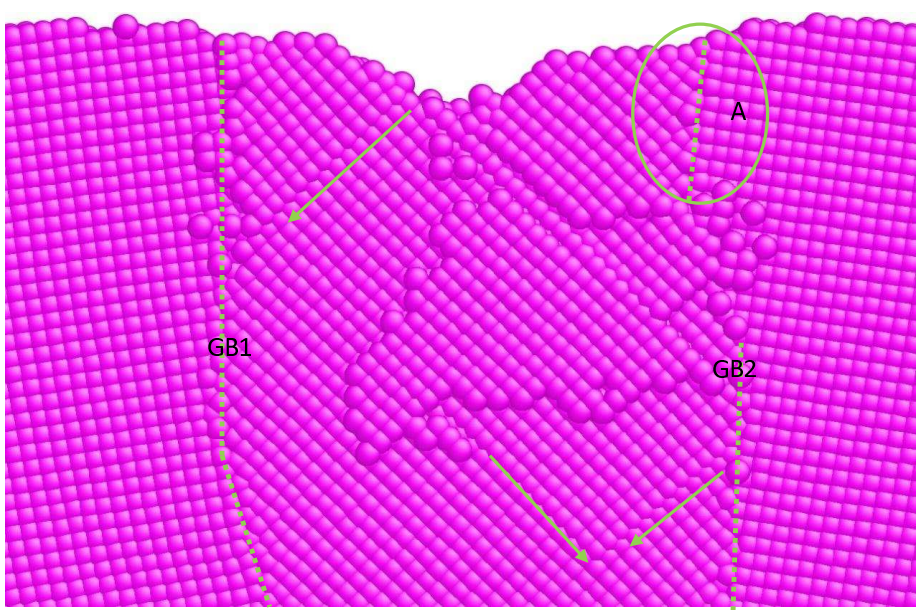

(c) $3 \mathrm{~nm}$

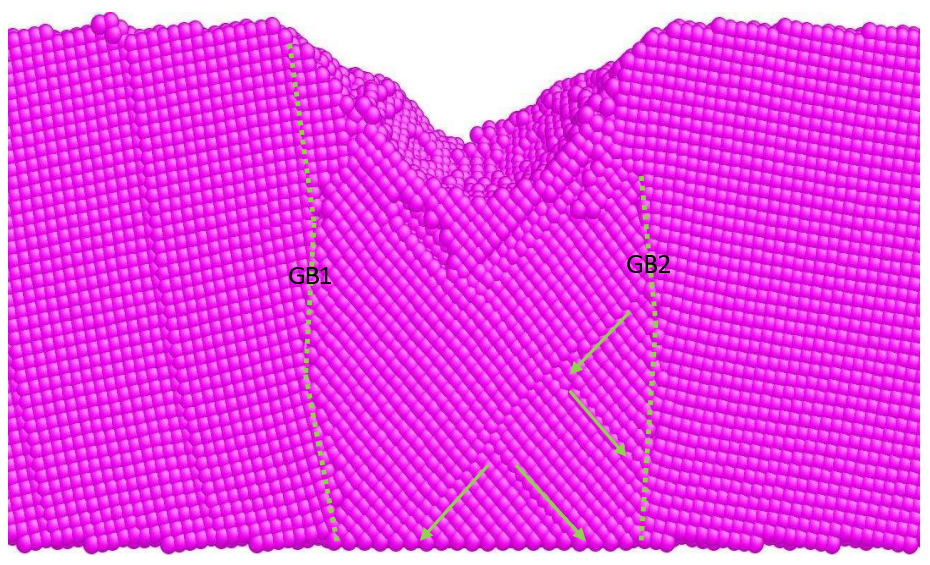

(d) $4 \mathrm{~nm}$

Fig. 5. (Continued)

see Fig. 5(c), the straight lines GB1 and GB2 become curves due to the large deformation occurred at G2 region. It should be noted that the line GB2 becomes obscure at region A marked with a circle, and there is a trend for both G2 and G3 to merge into one grain. As the indenter reaches at $4 \mathrm{~nm}$, both $\mathrm{G} 1$ and G2 at region B merge, and both G2 and G3 at region A also merge. Comparing Fig. 5(c) with $5(\mathrm{~d})$, the merging behavior of grains is observed clearly, it is due to the rotation deformation of the part region at grain G2. The result verifies that the transition of crystal structure occurs indeed.

Nanoindentation experiments have proved that near indentation site dislocations were observed. After successive indentations, two scanning tunneling 


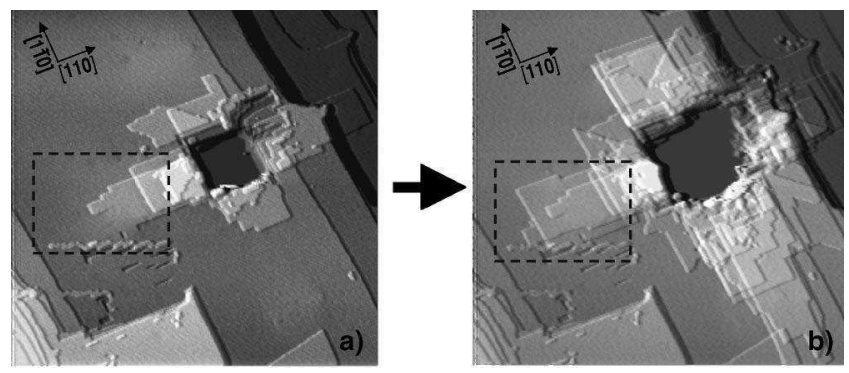

Fig. 6. Successive (a) and (b) nanoindentations on the same point of a $\mathrm{Au}$ (001) surface, the dislocations are identified near indenter in the dashed box.

microscope images of a surface of $\mathrm{Au}(100)$ were obtained by Carrasco et al. ${ }^{23}$ They set indenter at the same position, and images illustrate the redistribution of material around the indentation site, the material ejected during indentation "piles up" in a series of geometric terraces, and the termination points of some individual dislocations can be discerned. The geometry of such surface traces can be linked to the dislocation slip systems of the crystal. In the dashed box of Figs. 6(a) and $6(\mathrm{~b}),{ }^{23}$ a dislocation can be identified. Minor et al. ${ }^{24,25}$ developed a hybrid technique incorporating a nanoindenter into a transmission election microscope (TEM), providing in situ images of structural evolution beneath an indentation on $\mathrm{Al}^{25}$ where the initial contact with a dislocation-free grain can be observed, followed by the initiation of dislocation activity and the interaction of the dislocation structure with grain boundaries.

For the second example, the indenter is set at GB1. As the indenter reaches at $1 \mathrm{~nm}$, the slipping deformation is observed and it is originated from GB1, see arrow region at Fig. 7(a). As the indenter reaches at $2 \mathrm{~nm}$, the slipping deformations in several regions are observed, three of them are from GB1, one is from surface and another is from GB2, such as sites a, b, c, d and e, see Fig. 7(b). The result shows that both grain boundaries and surface are dislocation sources, the partial dislocation emitted from grain boundary and surface leading to the slipping deformation. As the indenter presses into at $3 \mathrm{~nm}$, the slipping deformation continues. It is observed that the crystal structure reverses the perfect lattice state at sites (a) and (d) due to dislocation slipping out of this local region. There may be no enough driving force, the following dislocation is not emitted from GB1, see Fig. 7(c). As the indenter reaches at $4 \mathrm{~nm}$, see Fig. $7(\mathrm{~d})$, more dislocations are emitted from GB1. The part line of GB1 disappears and the G1 and G2 merge in some regions. The transition of crystal structure is similar to the first example. Near the indenter and GB1, the deformation is big; Compared to GB1, the little deformation at GB2 is observed, and the line of GB2 is clear. It may be related to temperature distribution. One knows that the deformation is driven by applied load, and the influence of temperature on deformation and dislocation nucleation is important, and it should be considered. ${ }^{26-28}$ 
As a further study of the nature of defects generated during indentation, the temperature within the local zone under the pin tool is calculated for above two examples, respectively, and the zone is constructed around the pin tool and the distance from the tool is within $1.2 \mathrm{~nm}$. Figure 8 displays the curves of temperature versus the depth of indentation. The local temperature reaches over $600 \mathrm{~K}$ as the depth of indentation reaches about $4 \mathrm{~nm}$. Combining Figs. 4 and 7 with 8, it may be understood that temperature may be a vital for defects (such as amorphous and dislocations) nucleated in relatively higher temperature region. From Figs. 5-7, both simulations and experiment show that the dislocation nucleation or slip deformation is observed at the local zone near the indenter. The similar results by MD simulation are obtained by Chen et al. ${ }^{26}$ Rice and Beltz, ${ }^{27}$ Meyers and Chawla ${ }^{28}$ pointed out that the effects of thermal activation are very significant in lowering the load for dislocation nucleation, the tendency of the process of dislocation nucleation can be described by Arrhenius' equation. ${ }^{28}$ These analyses reveal that the nucleation of

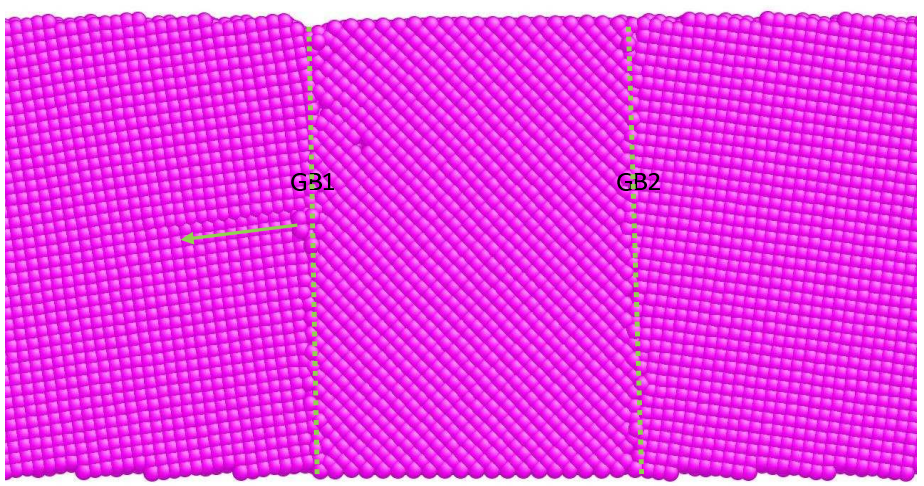

(a) $1 \mathrm{~nm}$

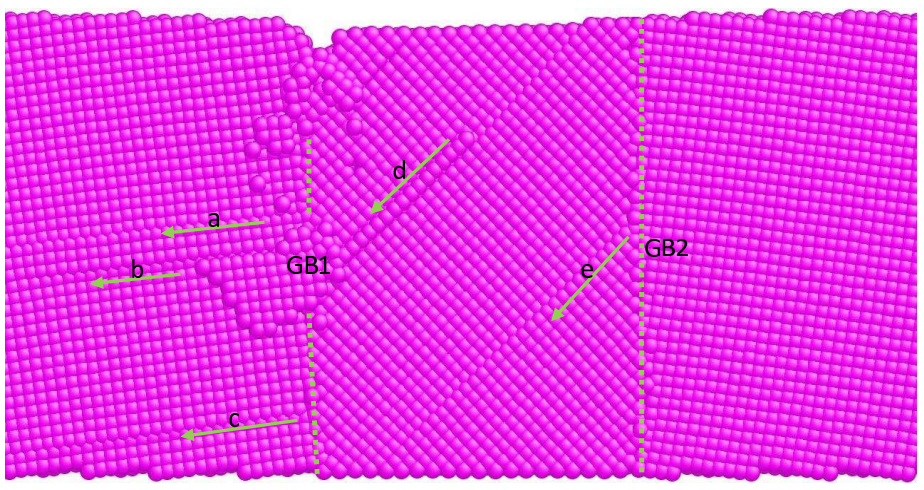

(b) $2 \mathrm{~nm}$

Fig. 7. Defects evolution during indentation for example 2: (a) $1.0 \mathrm{~nm}$, (b) $2.0 \mathrm{~nm}$, (c) $3.0 \mathrm{~nm}$, and (d) $4.0 \mathrm{~nm}$. 


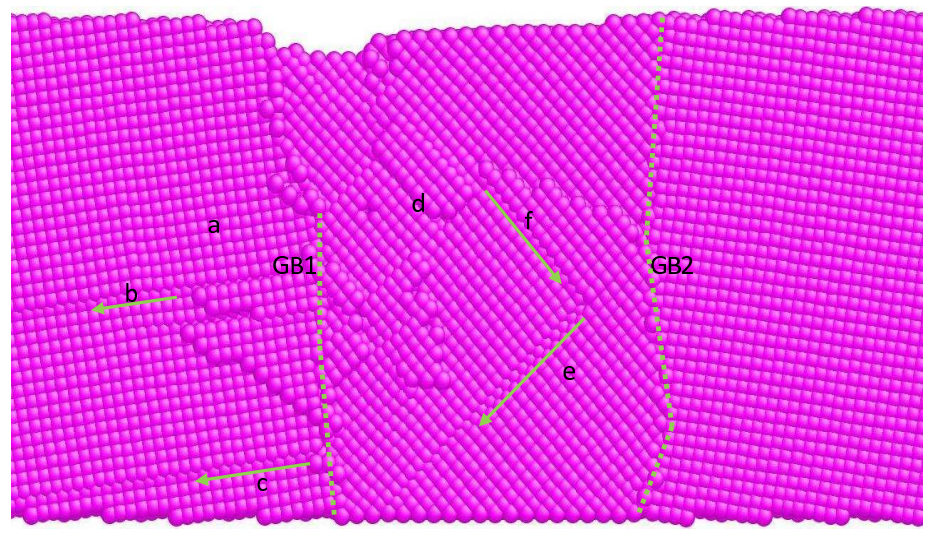

(c) $3 \mathrm{~nm}$

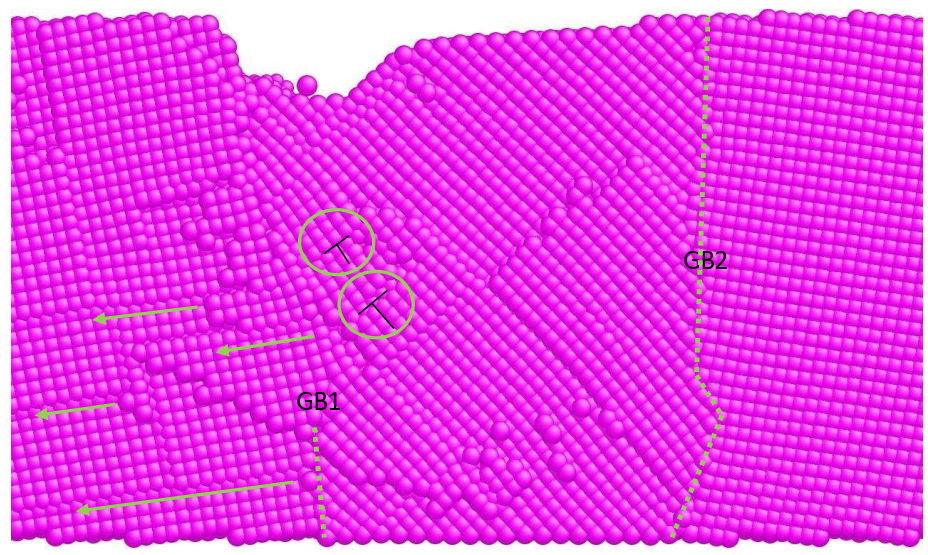

(d) $4 \mathrm{~nm}$

Fig. 7. (Continued)

surface dislocation is sensitive to temperature, and the applied load of dislocation nucleated is reduced with temperature increment.

In indentation experiment, direct temperature measurements are hindered by the localization and short duration of indentation. Figure 9 shows an atomic force microscope (AFM) image of the glassy alloy given by Kim et al. ${ }^{29}$ In this figure, discontinuous shear bands around an indent is observed. And further analysis of transmission electron microscope (TEM) image and selected area diffraction (SAD) pattern reveals clear diffraction spots associated with the presence of nanocrystallites. On the other hand, systematic experiments ${ }^{30}$ for the same glass alloy show that as the annealing temperature is up to $783 \mathrm{~K}$, partial crystallization could occur. It implies that a large rise in local temperature around the indent is possible, which may be to result from the adiabatic shear of metallic glass. ${ }^{31}$ Our simula- 


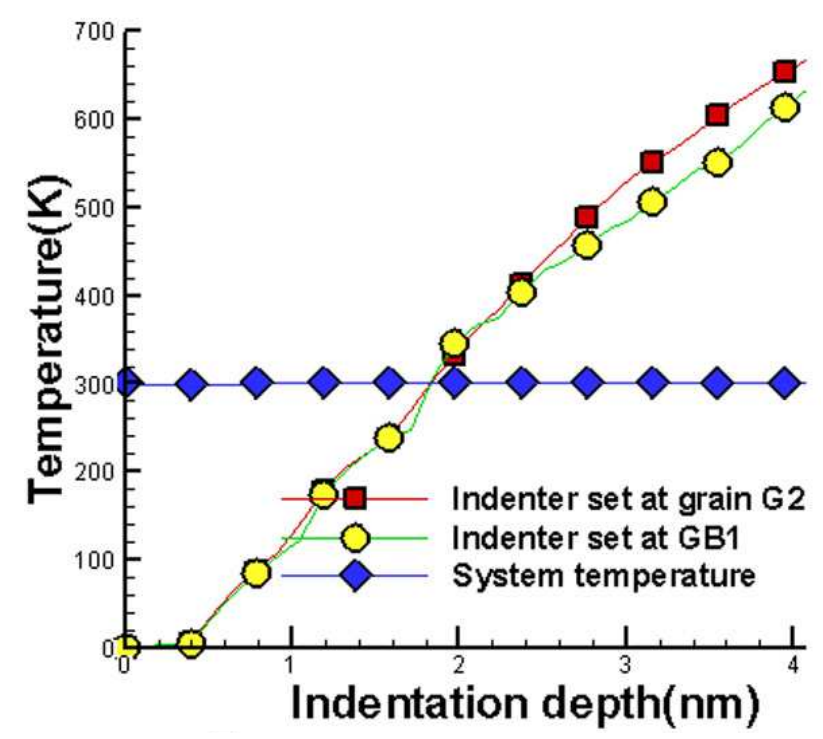

Fig. 8. Curves of temperature versus depth of indentation.

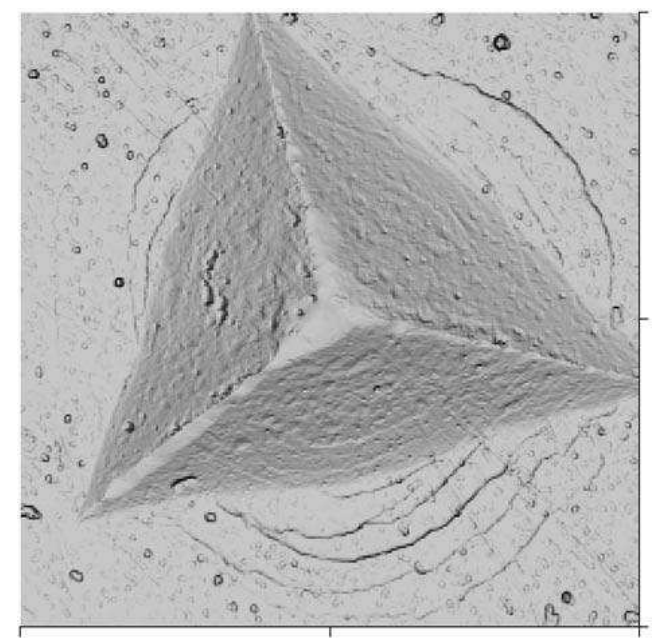

Fig. 9. AFM image of deformation around indent.

tion result of the high temperature distribution of local region around indenter is agreement with that of experimental observation and analysis.

In summary, MD simulations are carried out to understand the atomic scale mechanism of pin tool indentation of nc Ni. The simulation shows that the defects, such as dislocations or slipping deformation are near the tip. The local temperature near the tip is higher than that of system, and it has an important contribution on defects nucleation and evolution. 


\section{Acknowledgment}

The research presented here was supported by the National Natural Science Foundation of China (Grant no. 10721202, 10872197, 11021262).

\section{References}

1. X. D. Li et al., Nano Lett. 3, 1495 (2003).

2. X. D. Li, N. Wang and Q. H. Xiong, Nano Lett. 5, 1982 (2005).

3. U. Landman et al., Science 248, 454 (1990).

4. W. G. Hoover et al., Phys. Rev. A 42, 5844 (1990).

5. A. Gouldstone et al., Acta Materialia 55, 4015 (2007).

6. J. S. Kallman et al., Phys. Rev. B 47, 7705 (1993).

7. C. L. Kelchner, S. J. Plimpton and J. C. Hamilton, Phys. Rev. B 58, 11085 (1998).

8. A. Gouldstone, K. J. Van Vliet and S. Suresh, Nature 411, 656 (2001).

9. J. Li, A. H. W. Ngan and P. Gumbsch, Acta Materialia 51, 5711 (2003).

10. S. Ogata, J. Li and S. Yip, Science 298, 807 (2002).

11. D. M. Clatterbuck et al., Phys. Rev. Lett. 70, 104104 (2003).

12. X. L. Ma and W. Yang, Nanotechnology 14, 1208 (2003).

13. D. Feichtinger, P. M. Derlet and H. Van Swygenhoven, Phys. Rev. B 67, 024113 (2003).

14. K. J. Van Vliet, S. Tsikata and S. Suresh, Appl. Phys. Lett. 83, 1441 (2003).

15. A. F. Voter and S. P. Chen, Mat. Res. Symp. 82, 175 (1987).

16. H. Y. Jang et al., Mater. Sci. Forum 449, 89 (2004).

17. J. Diao et al., Acta Mater. 54, 643 (2006).

18. G. Z. Voronoi and A. Reine, Math 134, 199 (1908).

19. M. Parrinello and A. Rahman, J. Appl. Phys. 52, 7182 (1981).

20. S. Nose, Mol. Phys. 50, 1055 (1983).

21. W. G. Hoover, Phys. Rev. A 31, 1695 (1985).

22. A. R. Leach, Molecular Modelling: Principles and Applications (Addison-Wesley Longman, 1996).

23. E. Carrasco et al., Phys. Rev. B 68, 180102 (2003).

24. A. M. Minor et al., Appl. Phys. Lett. 79, 1625 (2001).

25. A. M. Minor et al., J. Mater. Res. 19, 176 (2004).

26. R. L. Chen et al., J. Appl. Phys. 104, 104907 (2008).

27. J. R. Rice and G. E. Beltz, J. Mech. Phys. Solids 42, 333 (1994).

28. M. A. Merers and K. K. Chawla, Mechanical Metallurgy (Prentice Hall Inc., US, 1983).

29. J. J. Kim et al., Science 295, 654 (2002).

30. J. G. Wang et al., J. Mater. Res. 15, 798 (2000).

31. H. J. Leamy, H. S. Chen and T. T. Wang, Metall. Trans. 3, 699 (1972). 